\title{
INDICADORES TECNOLÓGICOS SOBRE RECOBRIMENTOS PARA TUBOS METÁLICOS UTILIZADOS NA INDUSTRIA PETROQUÍMICA*
}

Bráulio Salumão de Oliveira ${ }^{1}$ Douglas Henrique Milanez ${ }^{2}$ Daniel Rodrigo Leiva ${ }^{3}$ Walter José Botta Filho ${ }^{4}$ Claudio Shyinti Kiminami ${ }^{5}$

\section{Resumo}

Este estudo avaliou os recentes desenvolvimentos em tecnologias para recobrimento de tubos metálicos utilizados na indústria petroquímica a partir de informações contidas em documentos de patentes depositadas entre os anos de 1996 e 2015. Destacaram-se novas composições para tintas e vernizes e processos de endurecimento e proteção à corrosão nos materiais metálicos. EUA, Japão e China figuram como os países que sediam o maior número de desenvolvimentos enquanto América do Norte, Europa e Ásia apontam como os principais mercados de interesse. No entanto, o estágio de desenvolvimento tecnológico sugere incerteza futura frente a novas soluções, sendo necessários estudos adicionais para confirmar as tendências observadas.

Palavras-chave: Tubos metálicos; Prospecção Tecnológica; Revestimento; Patente.

\section{TECHNOLOGICAL INDICATORS ON COATINGS FOR METALLIC PIPES USED IN THE PETROCHEMICAL INDUSTRY}

\section{Abstract}

This study evaluated recent developments on coating technologies for metallic pipes used in the petrochemical industry by analyzing information from patent documents filled between 1996 and 2015. New compositions for paints and varnishes as well hardening processes and corrosion protection of metallic materials highlighted. The USA, Japan and China are among the main filling countries while North America, Europe and Asia are the main markets. However, the technological development stage suggests uncertainty in the near future, consequently new studies are demanded to endorse all trends observed.

Keywords: Metallic pipes; Technological Forecasting; Coating; Patent.

1 Engenheiro de Materiais, Mestre em Ciência e Engenharia de Materiais, Doutorando, Programa de Pós-graduação em Ciência e Engenharia de Materiais, São Carlos, SP, Brasil

2 Engenheiro de Materiais, Doutor em Ciência e Engenharia de Materiais, Pós-Doutorando, Núcleo de Informação Tecnológica em Materiais, São Carlos, SP, Brasil

3 Engenheiro de Materiais, Doutor em Ciência e Engenharia de Materiais, Professor, Programa de Pós-graduação em Ciência e Engenharia de Materiais, São Carlos, SP, Brasil.

4 Engenheiro de Materiais, Doutor em Metalurgia e Ciência dos Materiais, Professor, Programa de Pós-graduação em Ciência e Engenharia de Materiais, São Carlos, SP, Brasil.

5 Engenheiro de Materiais, Doutor em Tecnologia de Fundição, Professor, Programa de Pósgraduação em Ciência e Engenharia de Materiais, São Carlos, SP. 


\section{INTRODUÇÃO}

O petróleo e o gás natural exercem grande impacto na economia global por figurarem atualmente, e com expectativa de se manterem por pelo menos mais 20 anos, como as principais fontes da matriz energética mundial [1-3]. Além de combustível, seus hidrocarbonetos derivados são fundamentais para a produção materiais utilizados em larga escala como plásticos, borrachas sintéticas, produtos farmacêuticos, cosméticos, etc. [4].

Produtos tubulares são amplamente utilizados em toda a cadeia petroquímica, desde a exploração e produção (tubos de perfuração, completação, condução, etc), etapas de refino (destiladores, trocadores de calor, etc.) até os dutos de transporte dos hidrocarbonetos para as refinarias e, delas, aos consumidores finais. São responsáveis por um custo significativo entre os equipamentos que compõem toda a cadeia e, em grande parte, este custo está associado aos materiais que os compõem e os processos necessários para atingir as propriedades requeridas de acordo com as especificações de projeto.

Os materiais metálicos, em especial os aços, são amplamente utilizados nesse setor por apresentarem boa relação entre propriedades mecânicas, físicas e custos, além de ampla disponibilidade [5]. Entretanto, desafios como aplicações em ambientes ácidos, altas pressões e/ou temperaturas e exposição à névoa salina implicam em dificuldades na seleção de materiais metálicos para essas aplicações.

Uma das maneiras de incrementar a resistência à corrosão, abrasão, e outras propriedades funcionais, que visam aumentar a vida útil dos tubos metálicos, é revestir esses produtos. Os revestimentos tendem a aumentar a vida útil dos produtos metálicos e possuem custo competitivo para uma vasta gama de aplicações quando comparados ao uso de materiais de alta liga. As soluções disponíveis para proteção dos metais podem ser classificadas de acordo com 0 mecanismo de atuação dos revestimentos como: formação de produtos insolúveis, por barreira, proteção catódica, entre outros [6].

Atualmente, as empresas produtoras de óleo e gás investem, em média, 0,4\% de sua receita de vendas em atividades de pesquisa e desenvolvimento, podendo chegar até 3,0\% para companhias de serviço [7]. Dado esse expressivo volume financeiro, o setor também é destaque no patenteamento, sendo responsável por cerca de 25 mil depósitos anuais no mundo [8,9]. Uma análise geral do patenteamento de tubos de aço utilizados na indústria petrolífera mostrou que os principais desafios tecnológicos do setor, após as propriedades de tração e impacto, como esperado, se referem a aplicação em ambientes com alto poder corrosivo [10].

Patentes são títulos de propriedade que permitem aos detentores o direito de exploração comercial exclusiva da tecnologia desenvolvida. Em contrapartida, o documento deve constar, em detalhes, o conteúdo técnico da matéria a ser protegida [11]. Indicadores tecnológicos podem ser elaborados a partir de informações contidas nos documentos de patentes. Entretanto, devido ao grande volume de informação geralmente encontrado referente a uma mesma área tecnológica, torna-se necessário utilizar softwares para tratamento da informação e cálculos estatísticos para auxiliar tal atividade [12].

Estudos baseados em indicadores tecnológicos são considerados estratégicos para organizações quem desejam compreender, manter ou ampliar seu poder de influência econômica e industrial baseados na exploração, produção, transporte e beneficiamento de hidrocarbonetos. No âmbito da Ciência e Engenharia de Materiais, em particular, as oportunidades figuram desde a seleção de materiais e 
processos menos onerosos até o desenvolvimento de novos produtos, com desempenho superior, que se tornam a vanguarda das soluções para os desafios técnicos do setor.

O objetivo deste estudo foi analisar os desenvolvimentos tecnológicos recentes em recobrimentos de materiais metálicos, em especial para proteção de tubos utilizados na indústria petroquímica. Em um primeiro momento, avaliou-se a evolução temporal dos processos de tratamento de superfície dos tubos. Em seguida, analisaram-se os indicadores para recobrimento dos tubos metálicos com foco para os países e regiões de origem das invenções e os mercados de interesse, o ciclo de desenvolvimento das tecnologias e os focos e as principais tendências de materiais e processos para esses novos produtos em classes de materiais.

\section{MATERIAIS E MÉTODOS}

Indicadores tecnológicos sobre tratamento de superfície de tubos utilizados pela indústria petroquímica foram levantados a partir do registro bibliográfico de documentos de patente indexados na base de dados Derwent Innovations Index. Os registros bibliográficos foram recuperados com base na seguinte expressão de busca: [TS=(petrochem* OR "crude oil*" OR petrol* OR "natural gas*" OR "chemical plant*" OR "chemical ind" oleochemic*) OR IP $\left.=\left(C 10^{*}\right)\right]$ AND [TS $=($ pip* OR tub* OR casing* OR liner* OR riser*) OR IP $=($ E21B-017* OR F16L-009/02 OR F16L-009/04 OR F16L-009/06 OR F16L-014* OR F16L-015* OR F16L-016/00 OR F16L-017* OR F16L-018* OR F16L-019* OR F16L-022*)] AND [IP=(B05* OR B32* OR C23* OR C25* OR $\left.{ }^{*} 30^{*}\right)$ ]. A expressão de busca contém palavras chave e códigos da Classificação Internacional de Patentes (CIP) para delinear a amostra de dados referente a indústria petroquímica e tubos. No caso de tratamento de superfície, utilizou-se apenas códigos da CIP categorizados como subdomínio tecnológico segundo o Observatório de Ciência e Tecnologia da França - OST [13], visto que os códigos CIP são atribuídos a uma patente a partir do conteúdo descrito nas reivindicações desses documentos, conferindo maior precisão para a amostra de dados recuperada. $O$ total de famílias de patente analisada foi 1.815 depositadas no período de 1996 a 2015.

Como o foco do presente estudo foram os recobrimentos de materiais metálicos, criou-se um subconjunto de dados com os documentos de patente filtrando os registros bibliográficos classificados com a CIP C23, que trata especificamente de tal temática, o que resultou num total de 541 famílias de patente no mesmo período.

A base de dados Derwent Innovations Index, disponível para acesso via Portal de Periódicos Capes, foi selecionada devido por cobrir mais de 40 escritórios regionais e nacionais de patentes; indexar famílias de patentes; ter controle sobre nomes das organizações que mais depositam patente no mundo; e melhorar os textos contidos nos registros bibliográficos (i.e. título e resumo), tornando a informação e a análise dos indicadores mais precisa [14].

Após a realização da busca na base, os registros bibliográficos foram coletados e armazenados num computador local. Em seguida, antes da análise estatística, a prioridade do primeiro depósito foi determinada utilizando o Earliest Priority Selector [15]. A análise foi conduzida com suporte do software Vantage Point (v. 7.1) e gráficos e mapas foram elaborados com suporte dos softwares Excel (v. 2010) e VOSviewer (v. 1.6.5), respectivamente. Os seguintes indicadores foram elaborados: (i) evolução temporal do patenteamento por tipo de processo de 
proteção anticorrosiva; (ii) mapeamento do mercado de tecnologias para recobrimento de materiais metálicos, por país; e (iii) ciclo de desenvolvimento tecnológico das tecnologias de recobrimento de materiais metálicos, conforme proposto por Mogee [16].

Alguns tratamentos foram realizados para elaboração dos indicadores finais. Os documentos de patente foram agrupados em períodos de quatro anos, para facilitar a análise da evolução temporal. Como uma patente pode ser depositada em um ou mais países, agrupou-se os países de depósito conforme sua macrorregião, enquanto que se manteve o país de origem para avaliar o mercado de tecnologias. Para elaboração do indicador sobre o ciclo de desenvolvimento tecnológico, foi necessário realizar a limpeza e padronização dos nomes das organizações (em especial das empresas) que são titulares das patentes, ou seja, detentoras do direito de explorá-las comercialmente. O mapa de co-ocorrência de códigos da CIP em nível de grupo voltados para materiais foram obtidos a partir dos agrupamentos denominados Química Macromolecular e Materiais-Metalurgia da OST [13]. As análises dos indicadores envolveram a comparação dos comportamentos observados nos indicadores e informações obtidas em outras fontes de informação, tais como publicações científicas, relatórios e dados técnicos.

\section{RESULTADOS E DISCUSSÃO}

\subsection{Panorama geral do patenteamento tratamentos de superfície de tubos para indústria petroquímica}

O número de documentos de patente sobre tratamentos de superfície em geral de tubos utilizados na indústria petroquímica tem crescido constantemente. Entre 1996 e 2014, esse aumento foi de 173\%, tratando-se de um reflexo direto dos interesses por tecnologias que atendam a demanda por produtos que se adequem ao ambiente altamente corrosivo e inóspito de operação das indústrias. Processos e aparatos para pintura líquida e tecnologias de recobrimento de materiais metálicos são os principais focos de desenvolvimento tecnológico nos últimos anos, conforme Figura 1. Processos eletrolíticos também têm recebido atenção quanto ao seu potencial de tratamento de superfícies de tubos enquanto que a fabricação via produtos em camada teve menos pedidos de patente nos últimos períodos da análise.

Uma análise detalhada dos dados sugere qual tem sido o foco de desenvolvimento dessas três abordagens de tratamento de superfície nos últimos anos. No caso de pintura líquida, o destaque em número de patentes era esperado devido à sua facilidade de aplicação e tradicional uso no setor. As tecnologias desenvolvidas nos últimos 20 anos têm se voltado para aparelhos de pulverização para descarga de líquidos ou outros materiais fluentes de duas ou mais fontes (como líquido e ar, pó e gás, etc.), com destaque para bocais, cabeçotes de pulverização ou outros escoadouros, com ou sem dispositivos auxiliares (por exemplo, válvulas e meios de aquecimento), além de outros acessórios. 




Fonte: Derwent Innovations Index. Elaborado pelos autores.

Figural 1. Evolução do número de depósitos de patente para diferentes tratamentos de superfície, por períodos.

Há também esforço para desenvolver aparelhos para descarga de líquidos sem mistura essencial com gás ou vapor (processos Airless), máquinas ou instalações para aplicação de líquidos ou outros materiais fluentes a superfícies de objetos ou efetuar outro trabalho por pulverização e meios para manipular ou segurar a peça. Esse foco deve-se a tendência atual de diminuição do uso de solventes em tintas, por questões ambientais e de rendimento. A aplicação de tintas $100 \%$ sólidos por volume, e seus processos de aplicação, figuram em destaque nesses desenvolvimentos por, além dos requisitos já citados, também apresentarem menor tempo de cura ao toque e final, resistência química, térmica além de maior dureza final do filme que incrementa a resistência à abrasão.

Para recobrimentos de materiais metálicos, as tecnologias buscam principalmente métodos que envolvem a difusão em estado sólido de apenas um elemento não metálico nas superfícies, tratamento químico da superfície dos tubos pela reação com um gás, depositando os produtos reacionais do material da superfície no revestimento (por exemplo, revestimentos de conversão e passivação de metais). Outras tecnologias buscam a proteção anódica, catódica ou utilizando-se de revestimento químico por decomposição, em que tanto os elementos químicos como soluções de elementos formadores de revestimento não deixam produtos reacionais do material da superfície no revestimento (depósito por contato). Há também patentes que reivindicam processos de limpeza ou retiradas de incrustações de materiais metálicos por meio de soluções salinas ou sais fundidos, com o objetivo de aumentar a vida útil dos revestimentos por impedir contaminação por sais ou outros elementos que diminuam a estabilidade dos filmes protetores. Por fim, no caso de processos eletrolíticos, destaca-se o recente esforço pela produção eletrolítica de compostos inorgânicos como tratamento de superfície de tubos.

\subsection{Análise das patentes em revestimento de materiais metálicos}

Como visto pela Figura 1, recobrimentos de tubos metálicos para indústria petroquímica têm recebido atenção quanto ao foco de desenvolvimento e uma análise mais detalhada desse conjunto de documentos de patente faz-se necessária. 
As tecnologias de recobrimento de tubos para indústria petroquímica têm se originado principalmente na China, EUA, Japão, Alemanha e França, como pode ser visto no mapa da Figura 2, que apresenta uma rede direcional entre os países de origem das patentes (nós em azul) com os mercados onde os depositantes protegem suas tecnologias. Em conjunto, tais países respondem por $83 \%$ do patenteamento mundial no tema da análise. Entretanto, os dados mostram que apenas a China e os EUA apresentam curvas de crescimento do número de depósitos nos últimos anos, o que sugere que organizações originárias destes dois países estão procurando novas tecnologias para atender aos desafios inerentes ao uso de recobrimentos em tubos metálicos para indústria petroquímica. Enquanto os EUA se destacam pelo patenteamento a partir de suas empresas, a China possui $22,5 \%$ de suas patentes originadas a partir de pesquisas em suas Universidades. Os demais países de origem das patentes aglomeram-se no mapa pelo principal mercado de interesse, porém possuem um baixo número de pedidos depositados (menos que cinco patentes).

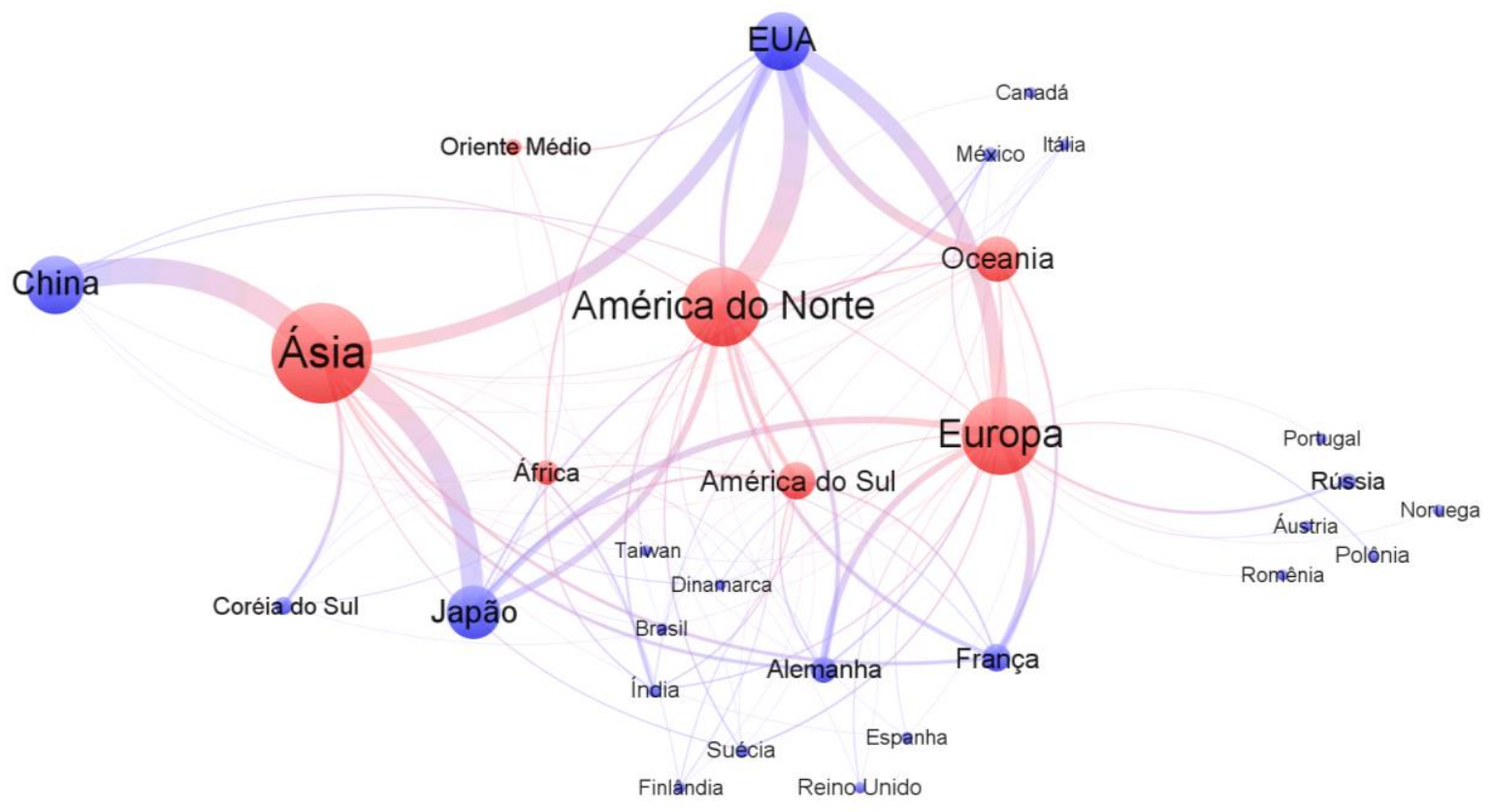

Fonte: Derwent Innovations Index. Elaborado pelos autores.

Figura 2. Países de origem das patentes (azul) e mercados (vermelho) onde estas patentes são depositadas. O tamanho do nó e a espessura das linhas são proporcionais ao total de documentos de patente.

Ásia (71\% das patentes), América do Norte (45\%) e Europa (43\%) são os principais mercados do depósito de patentes (nós em vermelho). Embora tais regiões possuam os principais países de origem das patentes, essa tendência mostra que os titulares procuram proteger suas tecnologias, sobretudo, em regiões onde os tubos são produzidos. O Oriente Médio, curiosamente, recebeu apenas $2,2 \%$ das patentes depositadas mundialmente, com origem, principalmente, dos EUA e do Japão. Apesar de figurarem como principais produtores de petróleo no mundo, boa parte do refino ocorre mais próximo aos maiores mercados consumidores, o que justifica a opção dos titulares em não estender o pedido de proteção até esses países.

As organizações americanas são as únicas que protegeram suas tecnologias nos vários mercados mundiais mapeados, com destaque para Ásia e Europa, além 
da América do Norte. O Japão procurou patentear, além da Ásia, na Europa, mas não na América do Norte, o que mostra que as empresas japonesas atuantes na produção de tubos para indústria petroquímica não necessariamente enxergam o mercado americano, canadense e mexicano como estratégicos. A China, por sua vez, confirma a tendência já observada em outros estudos de priorizar o patenteamento em seu próprio território, com pouca participação em sua extensão para outros mercados.

O desenvolvimento tecnológico em recobrimentos de materiais metálicos voltados para indústria petroquímica está em um estágio de crescimento, como indicado pela Figura 3. Tal tendência indica que está havendo investimento para solução dos desafios tecnológicos frente à produção de petróleo mundial e demandas da indústria petroquímica. No entanto, o posicionamento do último ponto gera incertezas quanto ao futuro dessas, visto que o decaimento da curva para os demais quadrantes indica cenários diferentes, caso o crescimento não continue (permanência no quadrante III).

Nos três possíveis cenários, haveria maturação tecnológica, mas caso, nos próximos anos, a atividade de patenteamento continue alta, mas haja diminuição no número de empresas que depositam tais patentes, o cenário seria de alta competição entre os agentes (quadrante II); por outro lado, caso não haja diminuição no número de empresas ativas e diminua o número de patentes, o cenário seria de abandono tecnológico (quadrante IV) - a solução para os desafios tecnológicos viria de outra abordagem; por fim, um decrescimento natural no número de patentes e de empresa significaria amadurecimento e morte da tecnologia (volta ao quadrante I) o cenário menos provável, dada a importância dos revestimentos da indústria petroquímica.

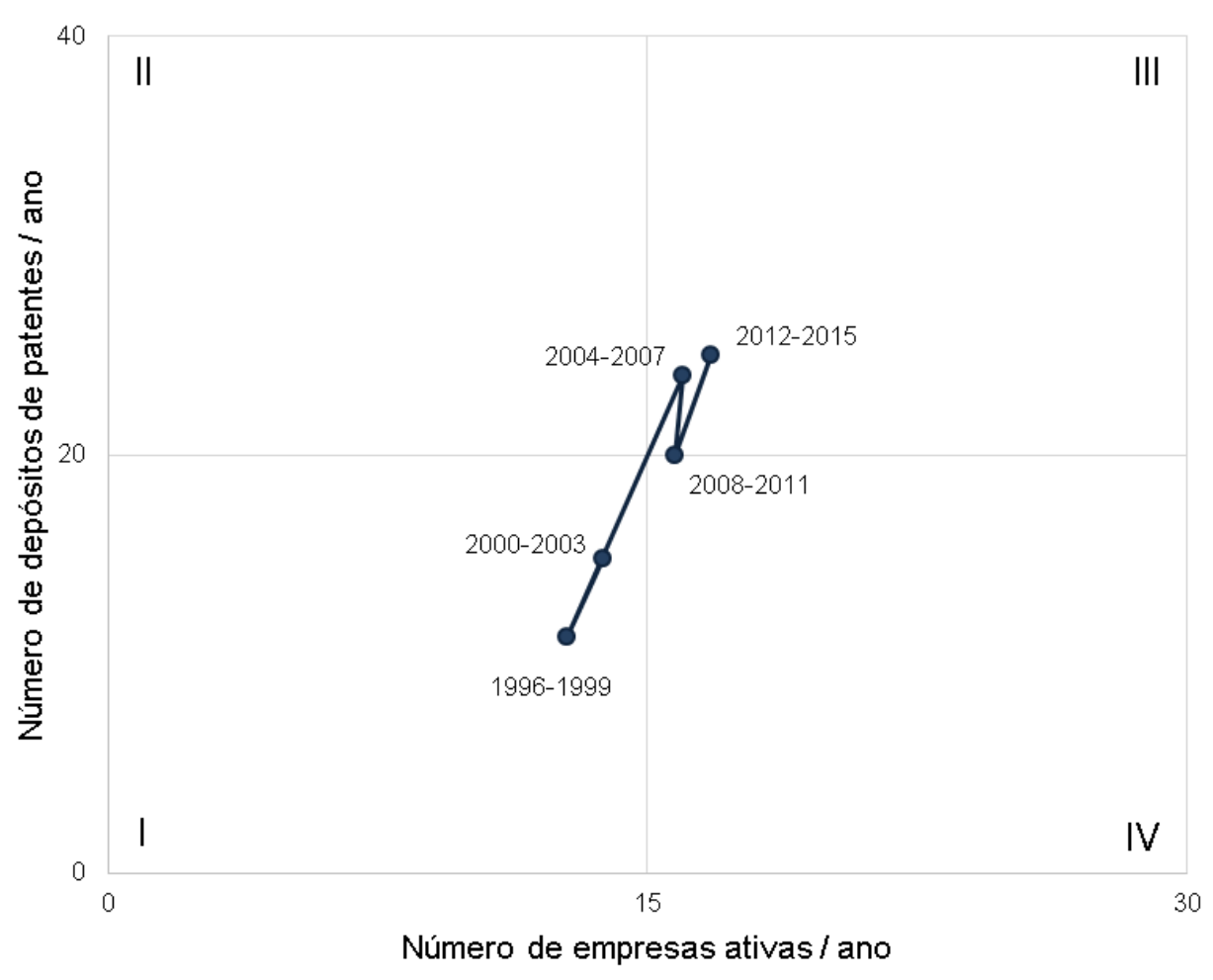

Fonte: Derwent Innovations Index. Elaborado pelos autores.

Figura 3. Ciclo de vida das tecnologias sobre recobrimento de tubos metálicos para aplicação na indústria petroquímica. 
A Figura 4 apresenta o mapa de co-ocorrência de códigos da CIP em nível de grupo para o conjunto de documentos de patente voltados para recobrimento de materiais metálicos. No caso, é possível verificar duas regiões distintas: à esquerda códigos da classificação que remonta a composições de tintas e acessórios para pintura; e à direita, ligas e processos de manufatura de materiais metálicos. Claramente, 0 desenvolvimento de novas tecnologias em materiais para recobrimento em tubos da indústria petroquímica tem focado em tintas e acessórios para pinturas.

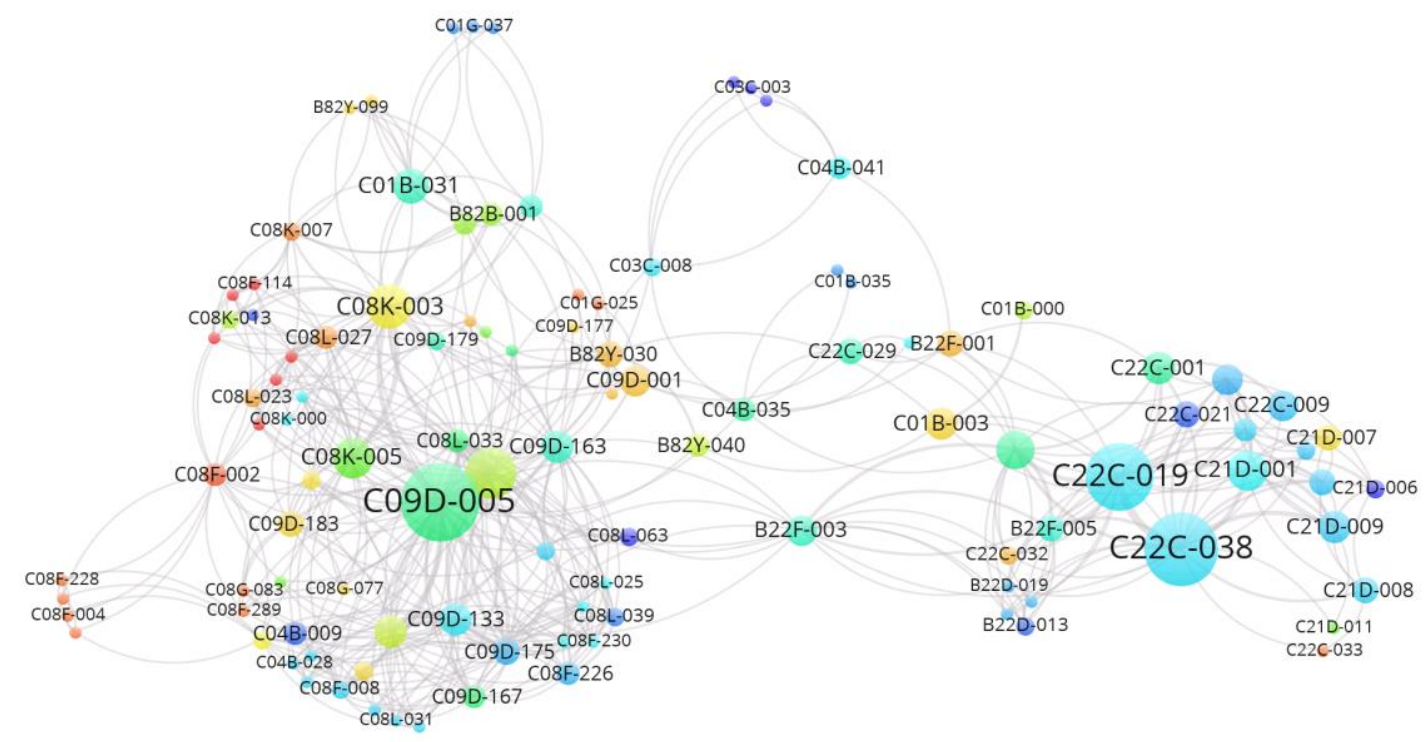

VOSviewer

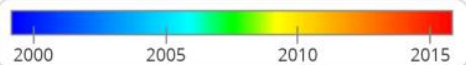

Fonte: Derwent Innovations Index. Elaborado pelos autores.

Figura 4: Mapa de co-ocorrência de códigos CIP voltados ao recobrimento de tubos metálicos utilizados na indústria petroquímica. O tamanho dos nós é proporcional ao número de documentos de patentes classificados com tal código CIP enquanto suas as cores mostram o ano médio em que o código da CIP aparece no conjunto de patentes, ou seja, quanto mais "quente" a cor, mais recente os documentos de patentes aos quais eles foram atribuídos.

Para tintas e acessórios para pintura, destaca-se a tendência de uso de composições de revestimentos de homopolímeros e copolímeros, não envolvendo anéis aromáticos na cadeia principal, com variadas terminações para tais cadeias, 0 que pode envolver um carboxílico (C22F-022), um halogênio (C08F-114 e C09D127), um anel carbocíclico aromático (C08F-212), um radical carboxila ou um sal do mesmo (C08F-220 e C08F-222), ou por uma ligação ao enxofre ou por um anel heterocíclico contendo enxofre (C08F-228). Há também tendência de tecnologias de tintas contendo polímeros obtidos por reações formando uma ligação éster carboxílicos de carbono na cadeia principal da macromolécula (C08G-063); ou obtidos por reações formando na cadeia principal da macromolécula uma ligação contendo silício, enxofre, nitrogênio, ou oxigênio (C08G-077 e C09D-183). Também se destacam composições de revestimento a base de lignina (C09D-197) e poliamidas (C09D-177). O uso de ingredientes inorgânicos (C08K-003) ou orgânicos não macromoleculares (C08K-005), nas mais variadas formas (C08K-007), podendo ser pré-tratadas (C08K-009), também tem sido alvo de desenvolvimento. 
A resina epóxi, embora contenha anéis aromáticos, também tem sido amplamente utilizada em tintas $100 \%$ sólidos por volume. Pigmentos para mistura de tintas (C09D-017) é outra tendência de desenvolvimento tecnológico pela necessidade de cura em menores intervalos de tempo espessantes e nivelantes para recobrimentos com apenas uma camada de tinta ou realizadas por um único passe, de maneira a aumentar a produtividade. Por fim, nanotecnologias (códigos classificados como B82B ou B82Y) também figuram entre as tendências recentes.

No caso de revestimentos metálicos, ligas a base de ferro (C22C-038) e ligas à base de níquel ou de cobalto (C22C-019) se sobressaem pela quantidade de documentos de patentes associados a tais códigos. No entanto, os desenvolvimentos tecnológicos mais recentes focaram o processamento metalúrgico do revestimento, com destaque para o uso de pós metálicos (códigos iniciados em $\mathrm{B} 22 \mathrm{~F}$ ), formação in situ de compostos para endurecimento de superfície (C22C032), modificação das propriedades físicas de ligas ferrosas por deformação (C21D007), controle do tratamento térmico (C21C-033) e combate a fragilização causada pela presença de hidrogênio (C01B-003).

\section{CONCLUSÃO}

Os indicadores tecnológicos elaborados a partir de documentos de patentes em recobrimentos de materiais metálicos utilizados em tubos na indústria petroquímica apontam para um aumento do número de depósitos no período entre os anos de 1996 e 2015. As áreas de maior crescimento foram pintura líquida, processos eletrolíticos e a classe específica de recobrimento de materiais metálicos. No caso de pintura líquida, que apresentou um maior número de documentos encontrados, os esforços de desenvolvimento se concentram em novas composições de tintas e vernizes com menor ou nenhuma adição de solventes, seus métodos de produção e instrumentos, dispositivos e acessórios de aplicação.

Em uma análise específica das patentes de recobrimento de materiais metálicos, observa-se que empresas dos EUA e do Japão e universidades da China concentram os depósitos de patentes no tema durante o período avaliado. Embora a China priorize o seu mercado interno, nota-se uma preferência geral dos EUA e Japão de extensão de depósitos para os mercados Europeu, Asiático e da América do Norte em primeiro plano, onde se concentra a maior parte da produção mundial de tubos metálicos utilizados na indústria petroquímica.

Apesar de apontar um caráter emergente no ciclo de desenvolvimento, o cenário tecnológico de recobrimento para tubos neste mercado é incerto. $O$ crescimento do número de depósitos é acompanhado também por um aumento do número de empresas que atuam no setor, o que sugere a busca por novas soluções frente aos desafios da aplicação.

Nota-se que as tecnologias para proteção dos tubos metálicos se dividem em dois grupos principais: ligas metálicas e polímeros. O segundo grupo apresenta uma tendência de desenvolvimentos mais recentes, pelo ano médio dos depósitos de patentes, especialmente em novas composições de tintas e vernizes. Já as tecnologias que partem dos materiais metálicos se encontram mais distribuídos em uma evolução temporal recente e seus temas de destaque se relacionam aos processos de obtenção de superfícies mais resistentes à abrasão e corrosão. 


\section{Agradecimentos}

Os autores agradecem o Núcleo de Informação Tecnológica em Materiais NIT/Materiais, o Programa de Pós-Graduação em Ciência e Engenharia de Materiais da UFSCar (PPGCEM-UFSCar), CAPES e FAPESP (Processos 2015/18878-8 e 2013/05987-8).

\section{REFERÊNCIAS}

1 IEA - International Energy Agency: World Energy Outlook 2014 - Executive Summary. OECD/IEA, Paris.

2 BP - Britsh Petroleum. BP Energy Outlook 2035.London, United Kingdom, January 2014.

3 EIA - Energy Information Administration - International Energy Outlook. Washington, D.C., United States, 2014.

4 THOMAS, J. E. Fundamentos de Engenharia de Petróleo. Interciência, 2004

5 PEREZ, T. E. Corrosion in the Oil and Gas Industry: An Increasing Challenge for Materials. Jom,[s.I.], v. 65, n. 8, p.1033-1042, 13 jul. 2013. Springer Science + Business Media. DOI: 10.1007/s11837-013-0675-3.

6 NUNES, L.P; LOBO, A.C O. Pintura industrial na proteção anticorrosiva. 4. ed. Rio de Janeiro: Interciência, 2012. 452 p.

7 STRATEGY + BUSINESS. $s+b$ Trend Watch: R\&D Investment Pays Off in Oil and Gas. Disponível em: <http://www.strategy-business.com/article/15201?gko=6930c >. Acesso em: 08 jul. 2015.

8 NORTON ROSE. Protecting Innovation in the Oil \& Gas Industry. 2012. Disponível em: $<$ http://www.nortonrosefulbright.com/files/protecting-innovation-in-the-oil--gas-industrypdf-195kb-63358.pdf>. Acesso em: 07 jul. 2015.

9 THOMSOM REUTERS. Oil, Gas \& Mining. 2015. Disponível em: $<$ http://stateofinnovation.thomsonreuters.com/industries/oil-gas-mining >. Acesso em: 06 maio 2015.

10 OLIVEIRA, B.S et al. Monitoramento tecnológico em ligas ferrosas utilizadas em tubos na indústria petrolífera a partir da análise de documentos de patentes. In: congresso anual da ABM - Internacional, 70., 2015, Rio de Janeiro. Anais... . São Paulo: Abm, 2015. p. 1 - 9.

11 INPI - Instituto Nacional da Propriedade Industrial. Guia Básico - Patentes. Disponível: http://www.inpi.gov.br/portal/artigo/guia_basico_patentes Acesso em: mai 2017.

12 FARIA, L.I.L.; GREGOLIN, J.A.R.; SANTOS, R.N.M. Technological Information And Materials Selection. Information Science for Decision Making. The International Journal of Information Science for Decision Making (Online), v. 1, p. 27-42, 1998.

13 OTS- Observatoire des Sciences et des Techniques. Science \& Technologies Indicateurs. Paris: Economica, 2010.

14 THOMPSON REUTERS. Derwent Innovations Index. Disponível em:<http://thomsonreuters.com/derwent-innovations-index/> Acesso em: mai 2017.

15 MILANEZ, D.H.; MILANEZ, M.G.; FARIA, L.I.L.; AMARAL, R.M.; GREGOLIN, J.A.R.. The Earliest Priority Selector for Compiling Patent Indicators. Proceedings of the 14th International Society of Scientometrics and Infometrics Conference - 2013 (ISSI '13). Vienna: Austria. p. 1950-1953.

16 MOGEE, M.E. Patents and technology intelligence. In: (Eds.) Keeping abreast of science and technology: technical intelligence for business. Columbus: Battelle Press, 1997. 